\title{
The dimensions of integral points and holomorphic curves on the complements of hyperplanes
}

by

\author{
Aaron Levin (Pisa)
}

1. Introduction. In this article we give a simple characterization of the possible dimensions of a set of integral points or a holomorphic curve on the complement of a union of hyperplanes in projective space. In Vojta's Nevanlinna-Diophantine dictionary [13], a holomorphic curve in a complex variety $V$, i.e., a holomorphic map $f: \mathbb{C} \rightarrow V$, corresponds to a set of integral points on $V$, assuming $V$ is defined over a number field. We therefore expect that the possible dimensions of a holomorphic curve and a set of integral points on $V$ will be the same, and indeed, we will see that this is the case when $V$ is the complement of a union of hyperplanes.

Our main theorems generalize a result of Evertse and Győry [4], who determined when all sets of integral points (over all number fields) on the complement of a union of hyperplanes are finite, and a result of $\mathrm{Ru}$ [11], who determined when all holomorphic maps to the complement of a union of hyperplanes are constant. As an application of our main theorems, we also generalize results of Fujimoto [6] and Green [7], who bounded the dimension of a holomorphic curve on the complement of hyperplanes in general position. Recently $\mathrm{Ru}$ and Ye [12] have found another use of our results, applying them in their proof of a certain big Picard theorem.

2. Results. If $\mathcal{H}$ is a set of hyperplanes on $\mathbb{P}^{n}$, we denote their union by $|\mathcal{H}|$. We denote by $\mathcal{L}$ a set of linear forms in $x_{0}, \ldots, x_{n}$ which define the hyperplanes in $\mathcal{H}$. We let $(\mathcal{L})$ be the complex vector space generated by the elements of $\mathcal{L}$.

Let $K$ be a number field and $M_{K}$ a complete set of inequivalent places of $K$. Let $S$ be a finite set of places in $M_{K}$ (which we will always assume contains the set $S_{\infty}$ of archimedean places of $\left.K\right)$. Let $\mathcal{O}_{K, S}$ denote the ring of

2000 Mathematics Subject Classification: Primary 11D57; Secondary 32H30, 11J97.

Key words and phrases: integral points, holomorphic curves, decomposable form equations. 
$S$-integers of $K$. If the set $|\mathcal{H}|$ is defined over $K$, we call a set $R \subset \mathbb{P}^{n}(K) \backslash|\mathcal{H}|$ a set of $S$-integral points on $\mathbb{P}^{n} \backslash|\mathcal{H}|$ if for every regular function $f$ on $\mathbb{P}^{n} \backslash|\mathcal{H}|$, defined over $K$, there exists $a \in K^{*}$ such that $a f(P) \in \mathcal{O}_{K, S}$ for all $P \in R$. Equivalently, $R$ is a set of $S$-integral points on $\mathbb{P}^{n} \backslash|\mathcal{H}|$ if there exists an affine embedding $\mathbb{P}^{n} \backslash|\mathcal{H}| \subset \mathbb{A}_{K}^{N}$ such that every $P \in R$ has $S$-integral coordinates.

For a set $R \subset \mathbb{P}^{n}$, we use $\operatorname{dim} R$ to denote the dimension of the Zariskiclosure of $R$ in $\mathbb{P}^{n}$. Similarly, for a holomorphic curve $f: \mathbb{C} \rightarrow \mathbb{P}^{n}$, we define $\operatorname{dim} f(\mathbb{C})$ to be the dimension of the Zariski-closure of $f(\mathbb{C})$ in $\mathbb{P}^{n}$.

We now state our two main theorems.

TheOREM 1A. Let $\mathcal{H}$ be a set of hyperplanes in $\mathbb{P}^{n}$ defined over a number field $k$. Let $\mathcal{L}$ be a corresponding set of linear forms. Let $m=\operatorname{dim} \bigcap_{H \in \mathcal{H}} H$. Then there exists a number field $K \supset k, S \subset M_{K}$, and a set $R$ of $S$-integral points on $\mathbb{P}^{n} \backslash|\mathcal{H}|$ with $\operatorname{dim} R=m+1$. Moreover, there exists a number field $K \supset k, S \subset M_{K}$, and a set $R$ of $S$-integral points on $\mathbb{P}^{n} \backslash|\mathcal{H}|$ with $\operatorname{dim} R=d>m+1$ if and only if there exists a partition of $\mathcal{L}$ into $d-m$ nonempty disjoint subsets $\mathcal{L}_{i}$,

$$
\mathcal{L}=\bigsqcup_{i=1}^{d-m} \mathcal{L}_{i}, \quad \mathcal{L}_{i} \neq \emptyset \quad \text { for all } i,
$$

such that

$$
\mathcal{L} \cap \sum_{j=1}^{d-m}\left(\left(\mathcal{L}_{j}\right) \cap\left(\mathcal{L} \backslash \mathcal{L}_{j}\right)\right)=\emptyset .
$$

Theorem 1B. Let $\mathcal{H}$ be a set of hyperplanes in $\mathbb{P}^{n}$. Let $\mathcal{L}$ be a corresponding set of linear forms. Let $m=\operatorname{dim} \bigcap_{H \in \mathcal{H}} H$. Then there exists a holomorphic map $f: \mathbb{C} \rightarrow \mathbb{P}^{n} \backslash|\mathcal{H}|$ with $\operatorname{dim} f(\mathbb{C})=m+1$. Moreover, there exists a holomorphic map $f: \mathbb{C} \rightarrow \mathbb{P}^{n} \backslash|\mathcal{H}|$ with $\operatorname{dim} f(\mathbb{C})=d>m+1$ if and only if there exists a partition of $\mathcal{L}$ into $d-m$ nonempty disjoint subsets $\mathcal{L}_{i}$ satisfying (1) and (2) above.

Note that in these theorems and elsewhere we define $\operatorname{dim} \emptyset=-1$. Given a partition of $\mathcal{L}$, one needs only elementary linear algebra to check (2). So running over all partitions of $\mathcal{L}$ and checking (2), we may determine the possible dimensions of a set of $S$-integral points on $\mathbb{P}^{n} \backslash|\mathcal{H}|$ (over all $K$ and $S$ ) or of a holomorphic map $f: \mathbb{C} \rightarrow \mathbb{P}^{n} \backslash|\mathcal{H}|$. This aspect of our theorem is not new, however. For instance, in the arithmetic case such an effective procedure is implicit in Laurent's theorem (see, e.g., [1, Th. 7.4.7] and its proof) where, if $\mathcal{L}=\left\{L_{0}, \ldots, L_{r}\right\}$, the set $\mathbb{P}^{n} \backslash|\mathcal{H}|$ is embedded in $\mathbb{G}_{m}^{r}$ by the map $P \mapsto\left(\frac{L_{1}}{L_{0}}(P), \ldots, \frac{L_{r}}{L_{0}}(P)\right)$. The simple characterizations in Theorems 1A and 1B have the advantage that they are very explicit and lend themselves readily to applications, as in Corollaries $3 \mathrm{~A}$ and $3 \mathrm{~B}$ or in the paper [12]. 
For our purposes, it is most convenient to use the language of integral points on varieties. However, we mention that our arithmetic results could also be stated in the more classical terminology of decomposable form equations, i.e., equations of the form

$$
F\left(x_{0}, \ldots, x_{m}\right) \in \beta \mathcal{O}_{K, S}^{*}, \quad\left(x_{0}, \ldots, x_{m}\right) \in \mathcal{O}_{K, S}^{m+1},
$$

where $S \subset M_{K}, \beta \in \mathcal{O}_{K, S}$ is nonzero, and $F \in K\left[x_{0}, \ldots, x_{m}\right]$ splits into a product of linear forms over some extension of $K$. Indeed, sets of integral points on complements of hyperplanes correspond, essentially, to sets of solutions to decomposable form equations. More precisely, let $Z$ be the closed subset of $\mathbb{P}^{m}$ defined by $F$. To each solution of (3), associate the projective point $\left(x_{0}, \ldots, x_{m}\right) \in \mathbb{P}^{m}$, and let $R \subset \mathbb{P}^{m}$ be the set of such points. Then $R$ is a set of $S$-integral points on $\mathbb{P}^{m} \backslash Z$. Note that, over some extension of $K, Z$ is a finite union of hyperplanes. Conversely, let $Z$ be a closed subset of $\mathbb{P}^{m}$, defined by a homogeneous polynomial $F \in K\left[x_{0}, \ldots, x_{m}\right]$, that is a finite union of hyperplanes over some extension of $K$. Let $R$ be a set of $S$-integral points on $\mathbb{P}^{m} \backslash Z$. Then it is easy to show that there exist a finite number of elements $\beta_{1}, \ldots, \beta_{r} \in \mathcal{O}_{K, S}$ such that each point $P \in R$ has a representative $\left(x_{0}, \ldots, x_{m}\right) \in \mathcal{O}_{K, S}^{m+1}$ that gives a solution to one of the decomposable form equations $F\left(x_{0}, \ldots, x_{m}\right) \in \beta_{i} \mathcal{O}_{K, S}^{*}, i=1, \ldots, r$.

There is an extensive literature on decomposable form equations. For instance, in a somewhat different direction from our results, it is known that the set of solutions to (3) can be naturally divided into a finite number of families, and the number of such families can be explicitly bounded (see $[3],[5],[8])$.

We will prove Theorems $1 \mathrm{~A}$ and $1 \mathrm{~B}$ in the next two sections. We now mention some of their consequences. The next two corollaries are immediate.

Corollary 2A (Evertse and Győry [4]). Let $\mathcal{H}$ be a set of hyperplanes in $\mathbb{P}^{n}$ defined over a number field $k$. Let $\mathcal{L}$ be a corresponding set of linear forms. Then all sets of $S$-integral points on $\mathbb{P}^{n} \backslash|\mathcal{H}|$ are finite for every number field $K \supset k$ and $S \subset M_{K}$ if and only if $\bigcap_{H \in \mathcal{H}} H=\emptyset$ and for every proper nonempty subset $\mathcal{L}_{1}$ of $\mathcal{L}$,

$$
\mathcal{L} \cap\left(\mathcal{L}_{1}\right) \cap\left(\mathcal{L} \backslash \mathcal{L}_{1}\right) \neq \emptyset .
$$

Corollary 2B $(\mathrm{Ru}[11])$. Let $\mathcal{H}$ be a set of hyperplanes in $\mathbb{P}^{n}$. Let $\mathcal{L}$ be a corresponding set of linear forms. Then all holomorphic maps $f$ : $\mathbb{C} \rightarrow \mathbb{P}^{n} \backslash|\mathcal{H}|$ are constant if and only if $\bigcap_{H \in \mathcal{H}} H=\emptyset$ and for every proper nonempty subset $\mathcal{L}_{1}$ of $\mathcal{L}$,

$$
\mathcal{L} \cap\left(\mathcal{L}_{1}\right) \cap\left(\mathcal{L} \backslash \mathcal{L}_{1}\right) \neq \emptyset .
$$

Let $[x]$ denote the greatest integer in $x$. 
Corollary 3A. Let $\mathcal{H}$ be a set of hyperplanes in $\mathbb{P}^{n}$ defined over a number field $k$. Suppose that the intersection of any $s+1$ distinct hyperplanes in $\mathcal{H}$ is empty. Let $r=\# \mathcal{H}$. Suppose $r>s$. Then for every number field $K \supset k$ and $S \subset M_{K}$, for all sets $R$ of $S$-integral points on $\mathbb{P}^{n} \backslash|\mathcal{H}|$,

$$
\operatorname{dim} R \leq\left[\frac{s}{r-s}\right]
$$

In particular, if $r>2 s$, then all such $R$ are finite. Furthermore, if the hyperplanes in $\mathcal{H}$ are in general position $(s=n)$, then the bound in (4) is achieved by some $R$.

Corollary 3B. Let $\mathcal{H}$ be a set of hyperplanes in $\mathbb{P}^{n}$. Suppose that the intersection of any $s+1$ distinct hyperplanes in $\mathcal{H}$ is empty. Let $r=\# \mathcal{H}$. Suppose $r>s$. Then for all holomorphic maps $f: \mathbb{C} \rightarrow \mathbb{P}^{n} \backslash|\mathcal{H}|$,

$$
\operatorname{dim} f(\mathbb{C}) \leq\left[\frac{s}{r-s}\right]
$$

In particular, if $r>2 s$, then all holomorphic maps $f: \mathbb{C} \rightarrow \mathbb{P}^{n} \backslash|\mathcal{H}|$ are constant. Furthermore, if the hyperplanes in $\mathcal{H}$ are in general position $(s=n)$, then the bound in (5) is achieved by some $f$.

Corollary 3B generalizes theorems of Fujimoto [6] and Green [7], who independently proved the case when the hyperplanes are in general position. Working in a different direction, Noguchi and Winkelmann [9] have generalized Fujimoto and Green's result (and its arithmetic analogue) to hypersurfaces of projective space in general position.

We now prove both corollaries simultaneously.

Proof of Corollaries $3 A$ and $3 B$. To prove (4) and (5), let $d>s /(r-s)$ be an integer. Let $\mathcal{L}=\bigsqcup_{i=1}^{d+1} \mathcal{L}_{i}$ be a partition of $\mathcal{L}$ into nonempty disjoint subsets. Note that since $r>s, \bigcap_{H \in \mathcal{H}} H=\emptyset$, so $m=-1$ in Theorems 1A and 1B. Using $d>s /(r-s)$, we see that there exists an index $i_{0}$ such that $\# \mathcal{L} \backslash \mathcal{L}_{i_{0}} \geq \frac{d}{d+1} r>s$. Since at most $s$ of the hyperplanes meet at a point, we therefore see that $\left(\mathcal{L} \backslash \mathcal{L}_{i_{0}}\right)$ is the whole $n+1$-dimensional vector space of linear forms, and so in particular,

$$
\mathcal{L} \cap \sum_{j=1}^{d+1}\left(\left(\mathcal{L}_{j}\right) \cap\left(\mathcal{L} \backslash \mathcal{L}_{j}\right)\right) \neq \emptyset .
$$

Thus, using Theorems 1A and 1B, we obtain the desired inequalities.

For the last assertions of the corollaries, where $s=n$, let $d=[n /(r-n)]$. Then we may partition $\mathcal{L}$ into nonempty disjoint subsets $\mathcal{L}_{i}, i=1, \ldots, d+1$, such that $\# \mathcal{L}_{i} \geq r-n$ for all $i$. Suppose that (2) does not hold. Let $L \in \mathcal{L}$ 
with

$$
L \in \sum_{j=1}^{d+1}\left(\left(\mathcal{L}_{j}\right) \cap\left(\mathcal{L} \backslash \mathcal{L}_{j}\right)\right) .
$$

For some index $i_{0}, L \in \mathcal{L}_{i_{0}}$. Note that

$$
\sum_{j=1}^{d+1}\left(\left(\mathcal{L}_{j}\right) \cap\left(\mathcal{L} \backslash \mathcal{L}_{j}\right)\right) \subset\left(\mathcal{L} \backslash \mathcal{L}_{i_{0}}\right),
$$

so that $L \in\left(\mathcal{L} \backslash \mathcal{L}_{i_{0}}\right)$. From $\# \mathcal{L}_{i_{0}} \geq r-n$, we have $\# \mathcal{L} \backslash \mathcal{L}_{i_{0}} \leq n$. This implies that the set $\mathcal{L} \backslash \mathcal{L}_{i_{0}} \cup\{L\}$ of at most $n+1$ linear forms is linearly dependent. This contradicts the hypothesis that the hyperplanes were in general position. Therefore (2) holds for this partition of $\mathcal{L}$, and so we are done by Theorems $1 \mathrm{~A}$ and $1 \mathrm{~B}$.

3. A reformulation of the problem. In this section we give a simple reformulation of the main problem. If $Y \not \subset|\mathcal{H}|$ is a linear subspace of $\mathbb{P}^{n}$ then we define

$$
\left.\mathcal{H}\right|_{Y}=\{H \cap Y \mid H \in \mathcal{H}\} .
$$

Note that $\left.\mathcal{H}\right|_{Y}$ may contain fewer hyperplanes than $\mathcal{H}$. We will denote by $\left.\mathcal{L}\right|_{Y}$ a set of linear forms defining the hyperplanes in $\left.\mathcal{H}\right|_{Y}$.

Consider the condition

(6) $Y \subset \mathbb{P}^{n}$ is a linear space, $Y \not \subset|\mathcal{H}|$, and $\left.\mathcal{L}\right|_{Y}$ is a linearly independent set.

We now reformulate our problem in terms of this condition.

TheOREM 4A. Let $\mathcal{H}$ be a set of hyperplanes in $\mathbb{P}^{n}$ defined over a number field $k$. Let $\mathcal{L}$ be a corresponding set of linear forms. There exists a number field $K \supset k, S \subset M_{K}$, and a set $R$ of $S$-integral points on $\mathbb{P}^{n} \backslash|\mathcal{H}|$ with $\operatorname{dim} R=d$ if and only if there exists a $Y$ satisfying (6) with $\operatorname{dim} Y=d$.

Theorem 4B. Let $\mathcal{H}$ be a set of hyperplanes in $\mathbb{P}^{n}$. Let $\mathcal{L}$ be a corresponding set of linear forms. There exists a holomorphic map $f: \mathbb{C} \rightarrow$ $\mathbb{P}^{n} \backslash|\mathcal{H}|$ with $\operatorname{dim} f(\mathbb{C})=d$ if and only if there exists a $Y$ satisfying (6) with $\operatorname{dim} Y=d$.

We will see that these theorems are simple consequences of the following two fundamental lemmas. We begin by giving the $S$-unit lemma, due to van der Poorten and Schlickewei [10] and Evertse [2].

Lemma 5A ( $S$-unit lemma). Let $k$ be a number field and let $n>1$ be an integer. Let $\Gamma$ be a finitely generated subgroup of $k^{*}$. Then all but finitely many solutions of the equation

$$
u_{0}+u_{1}+\cdots+u_{n}=1, \quad u_{i} \in \Gamma
$$

satisfy an equation of the form $\sum_{i \in I} u_{i}=0$, where $I$ is a subset of $\{0, \ldots, n\}$. 
The analytic analogue of the $S$-unit lemma is Borel's lemma.

Lemma 5B (Borel's lemma). Let $f_{1}, \ldots, f_{n}$ be entire functions without zeros on $\mathbb{C}$. Suppose that

$$
f_{1}+\cdots+f_{n}=1 .
$$

Then $f_{i}$ is constant for some $i$.

Lemma 6A. Let $\mathcal{H}$ be a set of hyperplanes in $\mathbb{P}^{n}$ defined over a number field $k$, and let $\mathcal{L}$ be a corresponding set of linear forms. All sets of $S$-integral points on $\mathbb{P}^{n} \backslash|\mathcal{H}|$ are non-Zariski-dense, for all choices of $K \supset k$ and $S \subset M_{K}$, if and only if $\mathcal{L}$ is a linearly dependent set. Furthermore, in this case any set $R$ of $S$-integral points on $\mathbb{P}^{n} \backslash|\mathcal{H}|$ is contained in a finite union of hyperplanes of $\mathbb{P}^{n}$.

Proof. Suppose $\mathcal{L}$ is a linearly dependent set. Let $\left\{L_{1}, \ldots, L_{m}\right\} \subset \mathcal{L}$ be a minimal linearly dependent subset, that is, no proper subset is linearly dependent. Then $\sum_{i=1}^{m-1} c_{i} L_{i}=c_{m} L_{m}$ for some choice of $c_{i} \in k^{*}$. Let $R$ be a set of $S$-integral points on $\mathbb{P}^{n} \backslash|\mathcal{H}|$. Since all of the poles of $L_{i} / L_{m}$ lie in $|\mathcal{H}|$, there exists an $a \in K^{*}$ such that $a L_{i} / L_{m}$ takes on $S$-integral values on $R$. Since the poles of $L_{m} / L_{i}$ also lie in $|\mathcal{H}|$, the same reasoning applies to $L_{m} / L_{i}$. Therefore $\frac{L_{i}}{L_{m}}(P)$ lies in only finitely many cosets of $\mathcal{O}_{K, S}^{*}$ for $P \in R$. By enlarging $S$, we may assume without loss of generality that $\frac{c_{i} L_{i}}{c_{m} L_{m}}(P)$ is an $S$-unit for all $P \in R$ and $i=1, \ldots, m$. Since

$$
\sum_{i=1}^{m-1} \frac{c_{i} L_{i}}{c_{m} L_{m}}(P)=1 \quad \text { for all } P \in R,
$$

by the $S$-unit lemma, it follows that all $P \in R$ either belong to one of the hyperplanes defined by $\sum_{i \in I} c_{i} L_{i}=0$ for some subset $I \subset\{1, \ldots, m-1\}$ (this equation is nontrivial by the minimality of the linear dependence relation) or they belong to a hyperplane defined by $\frac{c_{i} L_{i}}{c_{m} L_{m}}=t \in T$, where $T \subset \mathcal{O}_{K, S}^{*}$ is a finite subset containing the elements that appear in the exceptional solutions to the $S$-unit equation $\sum_{i=1}^{m-1} x_{i}=1$. Thus $R$ is contained in a finite union of hyperplanes of $\mathbb{P}^{n}$.

Conversely, suppose that $\mathcal{L}$ is a linearly independent set. After a $k$-linear change of coordinates, we may assume that $\mathcal{L}=\left\{x_{0}, \ldots, x_{m}\right\}$ for some $m \leq n$. Let $K \supset k$ be a number field with $\mathcal{O}_{K}^{*}$ infinite. Let $S$ be the set of archimedean places of $K$. Let $R$ be the set of points in $\mathbb{P}^{n}$ which have a representation where the coordinates are all $(S$-) units. Then it is easy to see that $R$ is a set of $S$-integral points on $\mathbb{P}^{n} \backslash|\mathcal{H}|$. We now show that $R$ is Zariski-dense in $\mathbb{P}^{n}$. This results from the $S$-unit lemma, or the following more elementary argument. Consider the set of homogeneous polynomials in $x_{0}, \ldots, x_{n}$ that vanish on $R \subset \mathbb{P}^{n}$. If this set is nonempty, let $p$ be a polynomial in this set with a minimal number of terms. Let $x_{i}$ be a variable 
that appears with different powers in two monomials of $p$ (clearly, $p$ has more than one term). Let $u \in \mathcal{O}_{K}^{*}$ be a unit that is not a root of unity. Let $q$ be the homogeneous polynomial obtained from $p$ by the substitution $x_{i} \mapsto u x_{i}$. Then $q$ also vanishes on $R$. By our choice of $u$ and $x_{i}, q$ is not a scalar multiple of $p$. However, $p$ and $q$ contain the same monomials. Therefore, there exists a linear combination of $p$ and $q$ that vanishes on $R$ and has strictly fewer terms than $p$. This contradicts the minimality of $p$, so $R$ is Zariski-dense in $\mathbb{P}^{n}$.

Lemma 6B. Let $\mathcal{H}$ be a set of hyperplanes in $\mathbb{P}^{n}$, and let $\mathcal{L}$ be a corresponding set of linear forms. There does not exist a holomorphic map $f: \mathbb{C} \rightarrow \mathbb{P}^{n} \backslash|\mathcal{H}|$ with Zariski-dense image if and only if $\mathcal{L}$ is a linearly dependent set. Furthermore, in this case all such holomorphic maps $f$ have image contained in a hyperplane of $\mathbb{P}^{n}$.

Proof. Suppose $\mathcal{L}$ is a linearly dependent set. Let $\left\{L_{1}, \ldots, L_{m}\right\} \subset \mathcal{L}$ be a minimal linearly dependent set. Then there exist nonzero constants $c_{1}, \ldots, c_{m-1}$ such that

$$
\sum_{i=1}^{m-1} c_{i} \frac{L_{i}}{L_{m}}=1 .
$$

Let $f: \mathbb{C} \rightarrow \mathbb{P}^{n} \backslash|\mathcal{H}|$ be a holomorphic map and let $g_{i}=\frac{L_{i}}{L_{m}} \circ f$. Then $g_{i}$ is an entire function without zeros on $\mathbb{C}$ since the image of $f$ omits $|\mathcal{H}|$. We also have $\sum_{i=1}^{m-1} c_{i} g_{i}=1$. By Borel's lemma, $g_{i}=\alpha$ for some $i$ and some constant $\alpha \in \mathbb{C}$. Therefore the image of $f$ is contained in the hyperplane defined by $L_{i}-\alpha L_{m}=0$.

Conversely, suppose that $\mathcal{L}$ is a linearly independent set. After a linear change of coordinates, we may assume that $\mathcal{L}=\left\{x_{0}, \ldots, x_{m}\right\}$ for some $m \leq n$. Let $f: \mathbb{C} \rightarrow \mathbb{P}^{n} \backslash|\mathcal{H}|$ be defined by $f=\left(1, e^{z}, e^{z^{2}}, \ldots, e^{z^{n}}\right)$. Looking at the growth as $z \rightarrow \infty$, it is clear that no homogeneous polynomial can vanish on $f(\mathbb{C})$, and so $f(\mathbb{C})$ is Zariski-dense in $\mathbb{P}^{n}$.

Theorems $4 \mathrm{~A}$ and $4 \mathrm{~B}$ now follow rather directly from Lemmas $6 \mathrm{~A}$ and 6B:

Proof of Theorems $4 A$ and $4 B$. We first make two general observations. In condition (6), even in the arithmetic case, we have allowed complex linear spaces $Y$. However, when the hyperplanes in $\mathcal{H}$ are defined over a number field $k$, it is easily seen that

$\max \{\operatorname{dim} Y \mid Y$ satisfies (6) $\}$ $=\max \{\operatorname{dim} Y \mid Y$ is defined over a number field and $Y$ satisfies (6) $\}$.

Secondly, if $Y$ satisfies (6), then for any $0 \leq d^{\prime} \leq \operatorname{dim} Y$, there exists a $Y^{\prime}$ satisfying (6) with $\operatorname{dim} Y^{\prime}=d^{\prime}$. To see this, let $\left.\mathcal{H}\right|_{Y}=\left\{H_{1}, \ldots, H_{r}\right\}$. If 
$r \geq \operatorname{dim} Y+1-d^{\prime}$, then let $Y^{\prime}$ be any linear space $Y^{\prime} \not \subset|\mathcal{H}|$ with

$$
\bigcap_{i=1}^{\operatorname{dim} Y+1-d^{\prime}} H_{i} \subset Y^{\prime}
$$

and $\operatorname{dim} Y^{\prime}=d^{\prime}$. Then $Y^{\prime}$ satisfies (6). If $r<\operatorname{dim} Y+1-d^{\prime}$, then $\bigcap_{i=1}^{r} H_{i}$ consists of a single linear space of dimension $\geq d^{\prime}$. Therefore, we may choose $Y^{\prime}$ to be a linear space $Y^{\prime} \not \subset|\mathcal{H}|$ with $\operatorname{dim} Y^{\prime}=d^{\prime}$ and $\left.\mathcal{H}\right|_{Y^{\prime}}$ consisting of a single hyperplane (ignoring the trivial case $d^{\prime}=0$ ).

Suppose now that there exists a $Y$ satisfying (6). Using the remarks above, we see that if the hyperplanes in $\mathcal{H}$ are defined over a number field, then there exists a $Y^{\prime}$ satisfying (6) with $Y^{\prime}$ defined over a number field and $\operatorname{dim} Y^{\prime}=\operatorname{dim} Y$. So, restricting things to $Y$ (or $Y^{\prime}$ ), it is immediate from Lemmas $6 \mathrm{~A}$ and $6 \mathrm{~B}$ that there exists a number field $K \supset k, S \subset M_{K}$, a set $R$ of $S$-integral points on $\mathbb{P}^{n} \backslash|\mathcal{H}|$ with $\operatorname{dim} R=\operatorname{dim} Y$, and a holomorphic map $f: \mathbb{C} \rightarrow \mathbb{P}^{n} \backslash|\mathcal{H}|$ with $\operatorname{dim} f(\mathbb{C})=\operatorname{dim} Y$.

Let $R$ be a set of $S$-integral points on $\mathbb{P}^{n} \backslash|\mathcal{H}|$ or the image of a holomorphic map $f: \mathbb{C} \rightarrow \mathbb{P}^{n} \backslash|\mathcal{H}|$. Repeatedly applying Lemma $6 \mathrm{~A}$ or $6 \mathrm{~B}$ (bearing in mind that when $\mathcal{L}$ is a linearly dependent set, $R$ is contained in a union of projective spaces to which the lemmas may be applied again), we see that there exists a $Y$ satisfying (6) with $\operatorname{dim} R \leq \operatorname{dim} Y$. By our earlier remarks, there then exists a $Y^{\prime}$ satisfying (6) with $\operatorname{dim} Y^{\prime}=\operatorname{dim} R$.

4. Proof of main theorems. We first make one more definition. We define the zero set of a set of linear forms $\mathcal{L}$ in $n+1$ variables to be the linear variety

$$
Z(\mathcal{L})=\left\{P \in \mathbb{P}^{n} \mid L(P)=0 \text { for all } L \in \mathcal{L}\right\} .
$$

Using Theorems $4 \mathrm{~A}$ and $4 \mathrm{~B}$ we are reduced to computing, for a given set of hyperplanes $\mathcal{H}$, the possible dimensions of a linear space $Y$ satisfying (6). Theorems $1 \mathrm{~A}$ and $1 \mathrm{~B}$ are therefore equivalent to the following theorem.

Theorem 7. Let $\mathcal{H}$ be a set of hyperplanes in $\mathbb{P}^{n}$. Let $\mathcal{L}$ be a corresponding set of linear forms. Let $m=\operatorname{dim} \bigcap_{H \in \mathcal{H}} H$. Then there exists a $Y$ satisfying (6) with $\operatorname{dim} Y=m+1$. Moreover, there exists a $Y$ satisfying (6) with $\operatorname{dim} Y=d>m+1$ if and only if there exists a partition of $\mathcal{L}$ into $d-m$ nonempty disjoint subsets $\mathcal{L}_{i}$ satisfying (1) and (2).

Proof. We first prove our assertion about the existence of a $Y$ satisfying (6) with $\operatorname{dim} Y=m+1$. If $m=-1$ this is trivial. Otherwise, we may take $Y$ to be any linear subspace of $\mathbb{P}^{n}$ of dimension $m+1$ with $\bigcap_{H \in \mathcal{H}} H \subset Y \not \subset|\mathcal{H}|$. In this case $\left.\mathcal{L}\right|_{Y}$ consists of a single linear form, which is therefore a linearly independent set. 
Suppose now that there exists a $Y$ satisfying (6) with $\operatorname{dim} Y=d>$ $m+1$. Let $m^{\prime}=\operatorname{dim} \bigcap_{\left.H^{\prime} \in \mathcal{H}\right|_{Y}} H^{\prime}$. Then $m^{\prime} \leq m$. Since $Y$ satisfies $(6),\left.\mathcal{H}\right|_{Y}$ consists of exactly $d-m^{\prime}$ hyperplanes of $Y$, say $H_{1}^{\prime}, \ldots, H_{d-m^{\prime}}^{\prime}$. Let $\mathcal{L}_{i}$, $i=1, \ldots, d-m^{\prime}$, consist of the linear forms in $\mathcal{L}$ that define hyperplanes which intersect $Y$ in $H_{i}^{\prime}$. Then we get a partition $\mathcal{L}=\bigsqcup_{i=1}^{d-m^{\prime}} \mathcal{L}_{i}$ of $\mathcal{L}$ into $d-m^{\prime}$ nonempty disjoint subsets. Let $j \in\left\{1, \ldots, d-m^{\prime}\right\}$. Then

$$
Z\left(\left(\mathcal{L} \backslash \mathcal{L}_{j}\right)\right) \supset \bigcap_{i \neq j} H_{i}^{\prime} .
$$

It follows from the linear independence of $\left.\mathcal{L}\right|_{Y}$ that $\bigcap_{i \neq j} H_{i}^{\prime}$ contains a point in $Y$ not contained in $H_{j}^{\prime}$. Therefore

$$
Z\left(\left(\mathcal{L}_{j}\right) \cap\left(\mathcal{L} \backslash \mathcal{L}_{j}\right)\right) \supset \operatorname{Span}\left(H_{j}^{\prime}, \bigcap_{i \neq j} H_{i}^{\prime}\right)=Y .
$$

So

$$
Z\left(\sum_{j=1}^{d-m^{\prime}}\left(\left(\mathcal{L}_{j}\right) \cap\left(\mathcal{L} \backslash \mathcal{L}_{j}\right)\right)\right) \supset Y
$$

Since $Y \not \subset|\mathcal{H}|$, we must therefore have $\mathcal{L} \cap \sum_{j=1}^{d-m^{\prime}}\left(\left(\mathcal{L}_{j}\right) \cap\left(\mathcal{L} \backslash \mathcal{L}_{j}\right)\right)=\emptyset$. Let $\mathcal{M}_{1}=\bigcup_{i=1}^{m+1-m^{\prime}} \mathcal{L}_{i}$ and $\mathcal{M}_{i}=\mathcal{L}_{i+m-m^{\prime}}$ for $i=2, \ldots, d-m$. Let $\mathcal{M}=$ $\bigcup_{i=1}^{d-m} \mathcal{M}_{i}$. It is straightforward to verify that

$$
\sum_{j=1}^{d-m}\left(\left(\mathcal{M}_{j}\right) \cap\left(\mathcal{M} \backslash \mathcal{M}_{j}\right)\right) \subset \sum_{j=1}^{d-m^{\prime}}\left(\left(\mathcal{L}_{j}\right) \cap\left(\mathcal{L} \backslash \mathcal{L}_{j}\right)\right) .
$$

Therefore (1) and (2) are satisfied (with $\mathcal{L}_{i}=\mathcal{M}_{i}$ ).

In the other direction, suppose that there exists a partition $\mathcal{L}=\bigsqcup_{i=1}^{d-m} \mathcal{L}_{i}$ into $d-m$ nonempty disjoint subsets such that (2) is satisfied. Let

$$
U_{0}=\sum_{j=1}^{d-m}\left(\left(\mathcal{L}_{j}\right) \cap\left(\mathcal{L} \backslash \mathcal{L}_{j}\right)\right) .
$$

We now define vector spaces $U_{i}, i=0, \ldots, d-m$, such that:

1. $U_{i} \subset U_{j}$ for $i<j$.

2. $\operatorname{dim} U_{i} \cap\left(\mathcal{L}_{i}\right)=\operatorname{dim}\left(\mathcal{L}_{i}\right)-1$ for $i>0$.

3. $U_{i} \cap \mathcal{L}=\emptyset$.

4. $U_{i}=\sum_{j=1}^{d-m}\left(U_{i} \cap\left(\mathcal{L}_{j}\right)\right)$.

Clearly, the space $U_{0}$ we have defined satisfies these conditions.

We now define $U_{i}$ inductively. Suppose that we have defined a $U_{i-1}$ with the above properties. Since $U_{i-1} \cap \mathcal{L}=\emptyset$, it follows that $U_{i-1} \cap\left(\mathcal{L}_{i}\right)$ is a 
proper subspace of $\left(\mathcal{L}_{i}\right)$. Therefore, since $\mathbb{C}$ is infinite,

$$
\bigcup_{L \in \mathcal{L}_{i}}\left(U_{i-1} \cap\left(\mathcal{L}_{i}\right)+L\right) \neq\left(\mathcal{L}_{i}\right) .
$$

So we easily see that we may add elements of $\left(\mathcal{L}_{i}\right)$ to $U_{i-1}$ to get a space $U_{i}$ with $\operatorname{dim} U_{i} \cap\left(\mathcal{L}_{i}\right)=\operatorname{dim}\left(\mathcal{L}_{i}\right)-1$ and $U_{i} \cap \mathcal{L}_{i}=\emptyset$. Also, we have $U_{i-1} \subset U_{i}$, and since we added only elements of $\left(\mathcal{L}_{i}\right)$, we deduce from the corresponding property of $U_{i-1}$ that $U_{i}=\sum_{j=1}^{d-m}\left(U_{i} \cap\left(\mathcal{L}_{j}\right)\right)$. In fact, we have

$$
U_{i}=U_{i} \cap\left(\mathcal{L}_{i}\right)+\sum_{j \neq i}\left(U_{i-1} \cap\left(\mathcal{L}_{j}\right)\right) .
$$

To show that $U_{i}$ has all the required properties, it only remains to show that $U_{i} \cap \mathcal{L}=\emptyset$.

Suppose that $L \in U_{i} \cap \mathcal{L}_{j^{\prime}}$ for some $j^{\prime}$. Since $U_{i} \cap \mathcal{L}_{i}=\emptyset$, we must have $j^{\prime} \neq i$. By (9), we may write $L=\sum_{j=1}^{d-m} u_{j}$, with $u_{j} \in\left(\mathcal{L}_{j}\right) \cap U_{i-1}$ for $j \neq i$ and $u_{i} \in\left(\mathcal{L}_{i}\right)$. Therefore $L-u_{j^{\prime}}=\sum_{j \neq j^{\prime}} u_{j}$ and

$$
L-u_{j^{\prime}} \in\left(\mathcal{L}_{j^{\prime}}\right) \cap\left(\mathcal{L} \backslash \mathcal{L}_{j^{\prime}}\right) .
$$

So $L-u_{j^{\prime}} \in U_{0} \subset U_{i-1}$. But $u_{j^{\prime}} \in U_{i-1}$, which implies that $L \in U_{i-1}$. This contradicts the assumption that $U_{i-1} \cap \mathcal{L}=\emptyset$. Therefore $U_{i} \cap \mathcal{L}=\emptyset$.

Let $U_{0}, \ldots, U_{d-m}$ be vector spaces defined as above. Let $Y=Z\left(U_{d-m}\right)$. We claim that $Y \not \subset|\mathcal{H}|,\left.\mathcal{L}\right|_{Y}$ is a linearly independent set, and $\operatorname{dim} Y=d$. Since $U_{d-m} \cap \mathcal{L}=\emptyset$, we have $Y \not \subset|\mathcal{H}|$. As $\operatorname{dim} U_{i} \cap\left(\mathcal{L}_{i}\right)=\operatorname{dim}\left(\mathcal{L}_{i}\right)-1$ for $i>0, U_{i} \subset U_{d-m}$ for all $i$, and $U_{d-m} \cap \mathcal{L}=\emptyset$, we must have

$$
\operatorname{dim} U_{d-m} \cap\left(\mathcal{L}_{i}\right)=\operatorname{dim}\left(\mathcal{L}_{i}\right)-1
$$

for all $i$. Therefore

$\operatorname{dim}\left(U_{d-m}+\left(\mathcal{L}_{i}\right)\right)=\operatorname{dim} U_{d-m}+\operatorname{dim}\left(\mathcal{L}_{i}\right)-\operatorname{dim} U_{d-m} \cap\left(\mathcal{L}_{i}\right)=1+\operatorname{dim} U_{d-m}$.

Let $\mathcal{H}_{i}$ be the set of hyperplanes defined by the elements of $\mathcal{L}_{i}$. The above equation shows that $\left.\mathcal{H}_{i}\right|_{Y}$ consists of a single hyperplane of $Y$. So $\left.\mathcal{H}\right|_{Y}$ consists of at most $d-m$ hyperplanes of $Y$. If $\operatorname{dim} Y=d$, then the facts that $\operatorname{dim} \bigcap_{\left.H \in H\right|_{Y}} H=m\left(\right.$ since $U_{d-m} \subset(\mathcal{L})$ ), and that $\left.\# \mathcal{H}\right|_{Y} \leq d-m$, imply that $\left.\mathcal{L}\right|_{Y}$ is a linearly independent set. So it remains to show that $\operatorname{dim} Y=d$, or equivalently, that $\operatorname{dim} U_{d-m}=n-d$. Repeatedly applying the equation $\operatorname{dim}(U+V)=\operatorname{dim} U+\operatorname{dim} V-\operatorname{dim} U \cap V$ we get

$$
\begin{aligned}
& \operatorname{dim} \sum_{i=1}^{d-m}\left(\mathcal{L}_{i}\right)=\operatorname{dim}(\mathcal{L})=n-m \\
&=\sum_{i=1}^{d-m} \operatorname{dim}\left(\mathcal{L}_{i}\right)-\sum_{j=1}^{d-m-1} \operatorname{dim}\left(\left(\mathcal{L}_{j+1}\right) \cap \sum_{i=1}^{j}\left(\mathcal{L}_{i}\right)\right)
\end{aligned}
$$


and

$$
\begin{aligned}
\operatorname{dim} U_{d-m}= & \operatorname{dim} \sum_{i=1}^{d-m}\left(\left(\mathcal{L}_{i}\right) \cap U_{d-m}\right) \\
= & \sum_{i=1}^{d-m} \operatorname{dim}\left(\mathcal{L}_{i}\right) \cap U_{d-m} \\
& \quad-\sum_{j=1}^{d-m-1} \operatorname{dim}\left(\left(\mathcal{L}_{j+1}\right) \cap U_{d-m} \cap \sum_{i=1}^{j}\left(U_{d-m} \cap\left(\mathcal{L}_{i}\right)\right)\right) .
\end{aligned}
$$

We claim that

$$
\left(\mathcal{L}_{j+1}\right) \cap U_{d-m} \cap \sum_{i=1}^{j}\left(U_{d-m} \cap\left(\mathcal{L}_{i}\right)\right)=\left(\mathcal{L}_{j+1}\right) \cap \sum_{i=1}^{j}\left(\mathcal{L}_{i}\right) .
$$

One inclusion is trivial. For the other, let $u \in\left(\mathcal{L}_{j+1}\right) \cap \sum_{i=1}^{j}\left(\mathcal{L}_{i}\right)$. This means that we have an equation $u=\sum_{i=1}^{j} u_{i}$ where $u \in\left(\mathcal{L}_{j+1}\right)$ and $u_{i} \in\left(\mathcal{L}_{i}\right)$. It follows easily from the definition of $U_{0}$ that $u, u_{1}, \ldots, u_{j} \in U_{0} \subset U_{d-m}$. The equation then follows. We also have $\operatorname{dim}\left(\mathcal{L}_{i}\right) \cap U_{d-m}=\operatorname{dim}\left(\mathcal{L}_{i}\right)-1$. Therefore, from (10) and (11), we get

$$
\begin{aligned}
\operatorname{dim} U_{d-m} & =\sum_{i=1}^{d-m} \operatorname{dim}\left(\mathcal{L}_{i}\right)-\sum_{j=1}^{d-m-1} \operatorname{dim}\left(\left(\mathcal{L}_{j+1}\right) \cap \sum_{i=1}^{j}\left(\mathcal{L}_{i}\right)\right)-(d-m) \\
& =n-m-(d-m)=n-d
\end{aligned}
$$

as was to be shown.

\section{References}

[1] E. Bombieri and W. Gubler, Heights in Diophantine Geometry, New Math. Monogr. 4, Cambridge Univ. Press, Cambridge, 2006.

[2] J.-H. Evertse, On sums of $S$-units and linear recurrences, Compos. Math. 53 (1984), $225-244$.

[3] —, The number of solutions of decomposable form equations, Invent. Math. 122 (1995), 559-601.

[4] J.-H. Evertse and K. Győry, Finiteness criteria for decomposable form equations, Acta Arith. 50 (1988), 357-379.

[5] - - - , The number of families of solutions of decomposable form equations, ibid. 80 (1997), 367-394.

[6] H. Fujimoto, Extensions of the big Picard's theorem, Tôhoku Math. J. (2) 24 (1972), 415-422.

[7] M. L. Green, Holomorphic maps into complex projective space omitting hyperplanes, Trans. Amer. Math. Soc. 169 (1972), 89-103.

[8] K. Györy, On the numbers of families of solutions of systems of decomposable form equations, Publ. Math. Debrecen 42 (1993), 65-101. 
A. Levin

[9] J. Noguchi and J. Winkelmann, Holomorphic curves and integral points off divisors, Math. Z. 239 (2002), 593-610.

[10] A. J. van der Poorten and H. P. Schlickewei, The growth condition for recurrence sequences, Macquarie Univ. Math. Rep. 82-0041 (1982).

[11] M. Ru, Geometric and arithmetic aspects of $P^{n}$ minus hyperplanes, Amer. J. Math. 117 (1995), 307-321.

[12] M. Ru and Y. Ye, A big Picard theorem for holomorphic maps into complex projective space, Canad. Math. Bull., to appear.

[13] P. Vojta, Diophantine Approximations and Value Distribution Theory, Lecture Notes in Math. 1239, Springer, Berlin, 1987.

Centro di Ricerca Matematica Ennio De Giorgi

Collegio Puteano

Scuola Normale Superiore

Piazza dei Cavalieri, 3

I-56100 Pisa, Italy

E-mail: aaron.levin@sns.it

Received on 20.8.2007

and in revised form on 12.4.2008 